\title{
Processing of conflicting and redundant stimulus information by pigeons
}

\author{
KUMIKO YOKOYAMA, DEENA DAILEY, and SHEILA CHASE \\ Hunter College of the City University of New York, New York
}

\begin{abstract}
Selective attention in processing of visual information by pigeons, trained on alternating sessions with two colors (red and green) and two forms (a diamond and an X shape) differentially associated with a left-right key choice task, was examined. A color and a form were presented together on probe trials during sessions in which, on other trials, only one of the dimensions, color or form, was shown. The dimension in effect on the surrounding trials had no influence on choice when the information provided by the two dimensions on probe trials was in conflict-color correct for one choice and form for the other. When both color and form redundantly cued the correct choice, there was no increase in accuracy in comparison with that associated with one dimension. Following separate training on the color and form discriminations, pigeons appeared to base their choices on color on some trials, on form on other trials, but not on both simultaneously. These findings are discussed in terms of an exemplar model of information processing.
\end{abstract}

Under different motivational states, in different environmental contexts, in the presence of different sequences of events, different properties of stimuli may control behavior. It is little wonder that the question of selective attention has, and continues to occupy, an important place in the study of learning and information processing. The situation examined here is one in which pigeons have information available from two stimulus dimensions (form and color) as cues indicating which of two responses (peck locations) will produce food. We asked how information is acquired when a discrimination between two colors and a discrimination between two forms that were trained separately is used. Would one or both cues be processed when they were redundant indicators of the correct choice? How would the pigeon respond when confronted with a situation in which the color and the form provided conflicting information as to which of the two choices was correct? Is response selection independent of the surrounding events or, as the literature on priming in visual search suggests, are choices influenced by recently encountered events?

Questions regarding the processing of the elements and compounds of visual stimuli have been addressed primarily by use of the matching-to-sample procedure. In matchingto-sample experiments, the sample is usually shown on the center of a row of three pecking keys, and the stimuli on the side keys determine which choice is correct on a given trial. Typically, after the pigeon pecks a visual stimulus, consisting of a single element or compound of two elements, the stimulus disappears and the pigeon is rewarded for choosing the

The research was made possible by PSC-CUNY Award 66328 to the third author. Correspondence concerning this article should be addressed to S. Chase, Department of Psychology, Hunter College, 695 Park Avenue, New York, NY 10021 (e-mail: schase@hunter.cuny.edu). key that matches the element, or one of the elements, in the compound. For example, in identity matching-to-sample, a pigeon may be trained to peck the red key following a peck at a red sample, and a green key following a peck at a green sample, and, on other trials, a vertical line following a peck at a vertical line and a horizontal line following a peck at a horizontal line. To test for attention to these elements, a compound composed of one element from each dimension - for example, a vertical line on a red background - is presented. Following pecks directed at this stimulus, the compound disappears, and, on some trials, one choice key is red and the other green, indicating this color as the dimension that is being tested on that trial. On other trials, the forms appear on the choice keys indicating that form is the relevant dimension. It is generally found that pigeons trained on two separate visual discrimination tasks perform better when each task is presented separately than when the separate elements are combined to form a compound. This element superiority effect occurs in both identity and conditional (nonidentity) matching-to-sample (Brown \& Morrison, 1990), even if element training is not given first (Grant \& MacDonald, 1986; Langley \& Riley, 1993), and it remains when exposure time is increased (Roberts \& Grant, 1978), as well as when the sample stimulus is visible when the choice is made (Grant \& MacDonald, 1986).

Attention to compounds and elements was examined in a different way in the present experiments. Instead of varying the appearance of the choice keys with their position irrelevant, the correct response was defined by the position of the keys, a white disk, either to the left or the right of the stimulus display - a procedure that decreases confusions between choices (D. S. Blough, 1996). Sessions of training with two colors as the discriminative stimuli (if red, peck the left key; if green, peck the right key), alternated with training on a task requiring a dis- 
crimination between two forms (if a diamond form, peck the left key; if an X form, peck the right key). The test stimuli, one of the elements from each dimension, were presented on probe trials within regular color or form training sessions. Both elements could be associated with the same choice, or each element could be associated with a different choice. In this situation, unlike in tests for attention using the matching-to-sample procedure, two elements associated with the same choice may act together, increasing the probability of making the correct choice. When the elements are experienced together, accuracy is better for the compound than for the elements (see, e.g., Chase \& Heinemann, 1972; Cook \& Wixted, 1997). In the present experiments, training on the two dimensions occurred on alternating sessions, and the elements were experienced together only on test trials. The question addressed here was whether two stimuli associated with the same choice, but not experienced together, would become associated. If so, as redundant cues to the correct choice, accuracy should be better for the compound than for either element alone.

The left-right position choice procedure also makes it possible to create test trials in which color is correct for one choice and form for the other - a situation in which the decision as to which choice to make may be made on the basis of color or of form, but not both. This provides an excellent opportunity to test the effects of recent events, the behavior reinforced on trials surrounding the test trials. The literature on priming suggests that recently encountered stimuli are more likely to be processed. For example, Pietrewicz and Kamil (1979) demonstrated that the ability to detect a particular prey type improves if it has been encountered on successive trials as opposed to when it is one of two prey types that have been encountered randomly on successive trials. In a similar study, using letters instead of images of moths, P. M. Blough (1991) found shorter reaction times for blocked trial sequences in which the same targets were successively presented than for mixed trial sequences in which more than one target appeared. In the present experiment, the pigeon's task was to decide whether to peck the left or right choice key rather than to search for a target. Of interest here was whether, in this somewhat different situation, choices would be based on color during color sessions and on form during form sessions.

Thus, the purpose of these experiments was to examine two phenomena known to influence attention to a stimulus complex in discrimination learning - the effects of training on processing of elements and compounds, and the effects of recently experienced events. Unlike in earlier experiments, in which the dependent measures of stimulus control were defined by the matching-to-sample procedure or by visual search, the correct choice was defined as a choice between two pecking keys that differed in position.

\section{GENERAL METHOD}

\section{Subjects}

The subjects were 4 White Carneaux pigeons (Columba livia) who had prior experience with the stimuli used in this experiment
(Schupak, 2002). The pigeons were maintained at $80 \%-85 \%$ of their free-feeding weights by supplementing food obtained during the experiment with Purina Gold pigeon pellets and mixed grain. Water and grit were freely available. They were housed in individual cages in a room on an 11:13-h light:dark cycle.

\section{Apparatus}

Two identical operant chambers were used. Each was divided into two compartments, with a rectangular opening separating the training chamber $(50.5 \mathrm{~cm}$ high $\times 45 \mathrm{~cm}$ wide $\times 32.5 \mathrm{~cm}$ deep $)$ from the compartment that contained a computer monitor. The training chamber was dimly illuminated by an overhead pilot light and ventilated by a fan. The monitor (Gateway 2000) was visible through a $32 \mathrm{~cm}$ high $\times 27 \mathrm{~cm}$ wide opening. A transparent touch screen (Elographics, Model E274) in front of the monitor was used to sense the location of the pigeons' pecks. Food pellets (Noyes, $45 \mathrm{mg}$ ) could be delivered into two small cups ( $7 \mathrm{~cm}$ long, $3 \mathrm{~cm}$ wide), one mounted at the left edge and the other at the right edge of the monitor opening, with the tops in line with the bottom edge of the opening. Food pellets were dispensed through tubes connected to rotary feeders on the top of the chamber. A Gateway 386 computer controlled experimental contingencies.

\section{Stimuli}

The pigeon's task was to peck on one of two white illuminated disks, $2 \mathrm{~cm}$ in diameter, that could appear at the left and right corners of the lower edge of the monitor opening, one near the left food cup and one near the right food cup. The centers of the disks were separated by $27 \mathrm{~cm}$. A peck at the left disk is referred to here as an R1 response, a peck at the right disk as an $\mathrm{R} 2$ response. For color training, the discriminative stimuli were a red or a green filled rectangle, $0.8 \mathrm{~cm}$ high $\times 0.4 \mathrm{~cm}$ wide. For form training, the discriminative stimuli were the outline of a diamond form or an X form composed of eight pixels that appeared white against the dark computer monitor. The forms were of the same size as the rectangles. R1 was defined as correct for both the red rectangle and the diamond form, and $\mathrm{R} 2$ was defined as correct for both the green rectangle and the $\mathrm{X}$ form. Test trials on which the color and form were correct for the same response, R1 or R2, will be referred to here as compatible test trials. Trials on which one response was correct for the color and the other for form will be referred to as incompatible test trials.

\section{Procedure}

The trial began with the appearance of one of the stimuli within the active area of the touch screen, a $12.5 \mathrm{~cm} \times 12.5 \mathrm{~cm}$ region within which a peck could be sensed. For 2 birds, $\mathrm{C}$ and $\mathrm{H}$, the stimuli always appeared in the center of the display; for another 2 birds, D and I, the stimuli could appear in any of 17 positions within this region. These two ways of presenting the stimuli were the training condition prior to the experiment (Schupak, 2002). When a peck within the active region occurred, the stimulus disappeared and two white disks (choice keys) appeared, one at the lower left corner and one at the lower right corner of the touch screen. The diagonal distance between the center of the active region and center of each choice disk was $16 \mathrm{~cm}$. After a correct response- a peck at the disk associated with the stimulus that had been shown-the luminance of the screen was increased to a light gray. If the correct response was R1, two food pellets were dropped into the left food cup. If the correct response was R2, two food pellets were dropped into the right food cup. After a 3-sec intertrial interval (ITI), the screen darkened, and the next stimulus was presented. When the incorrect disk was pecked, the computer monitor darkened for $4 \mathrm{sec}$, and the same stimulus was displayed again following the ITI. On such trials, following a peck within the active area, only the disk defined as correct for this stimulus appeared. Invariably this disk was pecked, and the next trial began after the ITI. Responses made on these correction trials were not used in data analyses. 


\section{EXPERIMENT 1}

The purpose of Experiment 1 was to determine which of two possible choices the pigeons would make when confronted for the first time with two elements - each from separately trained color and form discriminations-following alternating sessions in which only color or only form was relevant to the discrimination. Compatible and incompatible combinations were examined as well as whether the test stimuli were presented during color or form sessions.

\section{Method}

During training, a color or a form was presented within the active region of the display, marking the start of each trial. The pigeons were trained to discriminate between the red and green rectangles for 5 sessions followed by 36 sessions of training; this occurred on alternating days for the form and the color discriminations. The two colors and the two forms were presented equally often, in an otherwise random order, within each 136-trial session. In order to prepare for test trials during which either choice was followed only by the ITI, the percentage of reinforced trials for correct responses was reduced to $88 \%$ for 2 sessions and then to $76 \%$ for 3 additional sessions. Four test sessions followed, in which color and form sessions alternated. Each test session consisted of a random ordering of 104 training trials on which all correct responses were reinforced, 16 nonreinforced training trials, and presentation of the test stimuli on 16 nonreinforced probe trials. The test stimuli were composed of one of the colored rectangles and one of the forms presented side by side in the center of the computer monitor. There were four combinations of the color and form elements. For the compatible test trials, the colors and forms were associated with the same response - the red rectangle and the diamond form with $\mathrm{R} 1$, and the green rectangle and the X form with R2. For the incompatible test trials, the colors and forms were associated with responses that differed during training - the red rectangle with R1 and the X form with R2, the green rectangle with $\mathrm{R} 1$ and the diamond form with R2. For each of these four combinations, the colored rectangle appeared on the left and the form on the right and vice versa. For each of these eight configurations, the inner edges of the elements were separated by either 0.2 or $3 \mathrm{~cm}$.

\section{Results and Discussion}

As expected, because of their earlier training, accuracy was high for all of the pigeons during the 36 sessions of training that alternated between training on color and form. Accuracy on color (94\% correct) and on form (91\% correct) for the five final sessions preceding the test sessions did not differ significantly $[t(3)=0.836, p>.05]$.

Choice behavior on compatible test trials. On compatible test trials, the form and the color were associated with the same choice. Of interest was whether this would bind the elements on both dimensions into a single unit and thus enhance the probability of a correct choice (see, e.g., Chase \& Heinemann, 1972; Cook \& Wixted, 1997). There was no evidence of this. In fact accuracy was lower on the test trials than on the training trials. These data, averaged across the test sessions and the two separations of the test stimuli, are listed separately for color and form sessions for each bird in Table 1. The percentage of correct choices on trials on which both elements were associated with the same choice decreased significantly from training trials during color sessions, from $91 \%$ to $75 \%$ correct $[t(3)=8.094, p<.01]$, with a smaller, nonsignificant $[t(3)=0.538, p>.05]$ decrease in accuracy from $87 \%$ to $84 \%$ during form sessions. Latencies were about $0.5 \mathrm{sec}$ longer on test trials than on training trials.

Choice behavior on incompatible test trials. On incompatible test trials, one choice was correct for the color and the other was correct for the form. Because choices could not be treated as correct or incorrect on these trials, the percentages shown for the incompatible test trials in Table 1 are for choices that would have been correct

Table 1

Distribution of Choices During Form and Color Sessions

\begin{tabular}{|c|c|c|c|c|c|c|}
\hline \multirow[b]{2}{*}{ Bird } & \multicolumn{2}{|c|}{$\begin{array}{c}\text { Training Stimuli } \\
\text { Percentage Correct } \\
\end{array}$} & \multicolumn{2}{|c|}{$\begin{array}{c}\text { Compatible Test Stimuli } \\
\text { Percentage Correct }\end{array}$} & \multicolumn{2}{|c|}{$\begin{array}{l}\text { Incompatible Test Stimuli } \\
\text { Percentage Color Choices }\end{array}$} \\
\hline & $\begin{array}{l}\text { Form } \\
\text { Session }\end{array}$ & $\begin{array}{l}\text { Color } \\
\text { Session }\end{array}$ & $\begin{array}{c}\text { Form } \\
\text { Session }\end{array}$ & $\begin{array}{l}\text { Color } \\
\text { Session }\end{array}$ & $\begin{array}{c}\text { Form } \\
\text { Session }\end{array}$ & $\begin{array}{l}\text { Color } \\
\text { Session }\end{array}$ \\
\hline \multicolumn{7}{|c|}{ Experiment 1} \\
\hline $\mathrm{C}$ & 95 & 99 & 88 & 88 & 31 & 44 \\
\hline $\mathrm{D}$ & 96 & 95 & 81 & 75 & 44 & 31 \\
\hline I & 88 & 81 & 88 & 62 & 62 & 62 \\
\hline $\mathrm{H}$ & 70 & 90 & 81 & 75 & 69 & 50 \\
\hline Mean & 87 & 91 & 84 & 75 & 52 & 47 \\
\hline \multicolumn{7}{|c|}{ Experiment 2} \\
\hline $\mathrm{C}$ & 88 & 97 & 88 & 75 & 54 & 54 \\
\hline $\mathrm{D}$ & 94 & 97 & 100 & 92 & 96 & 83 \\
\hline I & 82 & 82 & 79 & 67 & 67 & 50 \\
\hline $\mathrm{H}$ & 82 & 92 & 96 & 88 & 92 & 79 \\
\hline Mean & 86 & 92 & 91 & 80 & 77 & 67 \\
\hline \multicolumn{7}{|c|}{ Experiment 3} \\
\hline $\mathrm{C}$ & 90 & 98 & 94 & 92 & 53 & 47 \\
\hline $\mathrm{D}$ & 88 & 97 & 94 & 97 & 58 & 61 \\
\hline I & 96 & 98 & 100 & 92 & 89 & 69 \\
\hline Mean & 91 & 98 & 96 & 94 & 67 & 59 \\
\hline
\end{tabular}


had the decision been based on color. Choices that would have been correct if based on form are the complement of these.

Given the data on priming and visual search, we expected choices on incompatible test trials to be based on the surrounding trials - that is, on the color during color sessions and on the form during form sessions. If choices had been based consistently on color during color sessions, and there were no errors due to inattention or confusion between the two colors, the data in the table would show $100 \%$ for color sessions and $0 \%$ for form sessions. Clearly, this was not the case. During color sessions, choices based on color occurred on $47 \%$ of the test trials (form on 53\%); during form sessions, choices based on color occurred on $52 \%$ of the test trials (form on $48 \%$ ). A one-way repeated measures ANOVA on the percentage of color choices in form and in color sessions indicated that the session type, the surrounding trials, did not have an effect on the dimension on which the decision was based $[F(1,3)=0.4576, p>.05]$.

These data, as well as the lack of improvement in accuracy when form and color redundantly cued the correct choice, suggest that the pigeons based their decisions on color on some trials and on form on others. However, it was possible that the approximately equal division of choices based on color and on form during incompatible test trials resulted from lack of attention to either dimension. This could have occurred if the change from one element on training trials to two on test trials was perceived by the pigeons as a completely new stimulus unrelated to the training conditions. In the following experiment, the potential effects of the change from one element in training to two in testing was reduced by training and testing with two elements.

\section{EXPERIMENT 2}

The similarity between the training and test conditions was increased by training the pigeons with two colors or two forms side by side prior to testing with a color and a form side by side. Only the smaller, $0.2-\mathrm{cm}$, spacing between the stimuli was used in this and subsequent experiments, in order to encourage viewing of the stimuli from both dimensions during testing.

\section{Method}

The subjects, apparatus, and stimuli were the same as those in Experiment 1 . Experiment 2 began approximately 60 days after the conclusion of Experiment 1. The procedure was the same as that used in Experiment 1, except that training trials began with the appearance of a pair of red or green rectangles presented side by side on color sessions and a pair of diamond or $\mathrm{X}$ forms presented side by side on form sessions. The pigeons were first trained for 12 sessions under the conditions in effect during Experiment 1. Eighteen alternating color and form sessions with pairs of colors or of forms followed. The percentage of reinforced trials for correct responses was then reduced to $88 \%$ for 4 sessions and then to $76 \%$ for 6 sessions in preparation for nonreinforced test trials. The test stimuli were the eight $0.2-\mathrm{cm}$ spaced combinations of form and color used in Experiment 1. Each test session consisted of 104 training trials with 32 probe trials ( 4 presentations of each of the 8 stimulus types) interspersed among the training trials. During the first 2 test sessions, 1 color and 1 form session, increased latency on probe trials suggested that the birds were beginning to discriminate between training and probe trials. To counteract this, and to eliminate possible effects on choice due to nonreinforcement, following 6 additional training sessions, the birds were given, on alternating days, 3 color and 3 form sessions with all correct responses reinforced on compatible trials and either choice reinforced on incompatible trials. With the introduction of this procedure, there were no obvious differences in latencies on test and training trials.

\section{Results and Discussion}

Reinforcement for correct choices and the increased similarity between the training and test stimuli was accompanied by a $6 \%$ increase in accuracy on compatible test trials in Experiment 2 in comparison with such trials in Experiment 1 (see Table 1). The differences in accuracy between training and compatible test stimuli during these sessions also decreased and were not statistically significant $(p<.05)$ for form $[t(3)=1.131]$ or for color $[t(3)=$ $2.697]$. During form sessions, the birds averaged $86 \%$ correct on training trials and $91 \%$ correct on test trials, and, during color sessions, these birds averaged $92 \%$ correct on training trials and $80 \%$ correct on test trials. As in Experiment 1 , there was no evidence of improvement when both stimuli were associated with the same choice. The possibility that this potential for improvement was offset in Experiment 1 by decreased attention on test trials was eliminated in this experiment by providing reinforcement on test trials and decreasing the difference in the appearance of the test and training stimuli.

These findings suggest that the pigeons based their choices on one of the two elements. On compatible trials, because both elements are associated with the same key choice, it is impossible to tell whether the choice was based on color, or on form, or on color on some trials and on form on others. Choices on incompatible test trials provide an answer. Examination of the percentages of choices based on color on incompatible test trials (see Table 1) suggests that during Experiment 2, responses were divided between form and color with a bias toward choices based on color, a bias that might reflect the slightly greater accuracy on color training trials than on form training trials.

Once again, the findings of Experiment 1, that the division of responses between color and form on incompatible test trials was not influenced by the type of surrounding trials, was confirmed by anOVA $[F(1,3)=8.33, p>$ $.05]$, here under conditions in which the probability of inattention was reduced by increasing the similarity between test and training trials. That the failure to obtain an effect of session type was due to inattention on incompatible test trials was examined further in Experiment 3 by a direct test of this possibility.

\section{EXPERIMENT 3}

In the previous experiments, on test trials one color and one form were shown. This balance was destroyed 
in Experiment 3 by use of test stimuli made up of three elements: two colors and one form, or one color and two forms. If, as Honig and Stewart (1989) and Emmerton (2001), among others, have shown, pigeons are sensitive to the number of items in an array, there should be an increase in the percentage of choices based on the more frequent element in the array. On incompatible test trials, there should be a bias toward choices based on color in the presence of two colors and one form and a bias toward choices based on form in the presence of two forms and one color. If the test stimuli on these trials were not processed - that is, if the pigeons were not attending to the stimuli on test trials - creation of this inequality between the number of forms and the number of colors should have no effect. Our previous findings suggested that this should be independent of session type.

\section{Method}

Because Bird H became ill prior to the start of the experiment, data were obtained only from the remaining 3 pigeons. The apparatus and the procedure were the same as in the previous experiments, except that the stimuli were composed of three elements. In training, these were three red or green rectangles for color sessions and three diamond forms or $\mathrm{X}$ forms for form sessions. Adjacent elements were separated by $0.2 \mathrm{~cm}$. Following 12 sessions in which training on color and form alternated, the probe trials were presented during three color sessions that alternated with three form test sessions. For the test trials on which the number of color elements exceeded the number of form elements, there was an $\mathrm{X}$ form with two green or red rectangles, or a diamond form with two green or red rectangles. For trials on which the number of form elements exceeded the number of color elements, there was a red rectangle with two diamond or $\mathrm{X}$ forms, or a green rectangle with two diamond or $\mathrm{X}$ forms. As in Experiments 1 and 2, there were four combinations of elements, two with compatible combinations of stimuli and two with incompatible combinations of stimuli. For each of these four basic combinations, the odd element was in the center, on the left, or on the right. This yielded six different configurations for each of the four combinations. Each of these 24 test stimuli was presented once in each session, interspersed among 112 color or form training trials. As was true for the final 6 test sessions in Experiment 2, all correct responses were reinforced in the presence of the compatible test stimuli, and either response was reinforced in the presence of the incompatible test stimuli.

\section{Results and Discussion}

Under the conditions in effect in Experiment 3, the differences in accuracy between the training and the compatible test stimuli virtually disappeared (see Table 1). Once again, we failed to find an increase in accuracy on compatible test trials, confirming our earlier suggestion that only one of the elements was processed. The percentage of responses based on color did not differ significantly as a function of session type $[F(1,2)=1.344, p>.05]$, replicating the findings of the previous experiments.

Although the surrounding events, color or form session, did not have an effect on the dimension on which the choice was based, changing the combination of elements did. Having two elements of the same type, color or form, biased responding toward the dimension that was represented by two forms or two colors as revealed by the division of choices on incompatible test trials (see Table 2). All birds made more choices in the presence of the major element during both form and color sessions, significantly so for Birds C and D. Evidence that the pigeons were sensitive to the number of elements on incompatible test trials decreased the possibility that the failure to find an effect of session type in the three experiments was due to failure to attend to the stimuli on these trials.

\section{GENERAL DISCUSSION}

In these experiments, we examined processing of visual information when elements from two separately trained discriminations were combined. Unexpectedly, surrounding trials had no influence on which dimension was processed when the two elements of the compound, a form and a color, were associated with different choices. However, our procedure was quite different from the search situation in which priming by a consistent sequence of events is observed (P. M. Blough, 1991; Pietrewicz \& Kamil, 1979). Different processes may be involved in visual search and in discrimination tasks (D. S. Blough, 2002). The conditions under which the sequence of events does, or does not, have an effect on a choice discrimination remain to be defined.

Unlike the matching-to-sample procedure used in studies of selective attention, in which on test trials only one dimension of the compound is relevant, the present experiments enabled us to observe the effects on choice accuracy when more than one dimension of a compound stimulus was relevant. Although the pigeons clearly learned to associate each color and each form with the appropriate choice keys, when tested with the two elements associated with the same choice key, there was no increase in accuracy. This finding contrasts with choice situations in which there is an increase in accuracy in the presence of a compound as opposed to its elements. In a review of the literature on combination laws in conditioning with compound stimuli, Kehoe and Gormezano (1980) noted that "as the data accumulate, the theories appear to be converg-

Table 2

Effect of Number of Elements on Choices on Incompatible Probe Trials

\begin{tabular}{|c|c|c|c|c|c|c|}
\hline \multirow[b]{2}{*}{ Bird } & \multicolumn{2}{|c|}{ No. Form Choices } & \multicolumn{2}{|c|}{ No. Color Choices } & \multirow{2}{*}{$\begin{array}{c}\% \text { Choices } \\
\text { Based on } \\
\text { Major Element }\end{array}$} & \multirow{2}{*}{$\begin{array}{c}\text { Probability } \\
\text { Due to Chance }\end{array}$} \\
\hline & 2 & 1 & 2 & 1 & & \\
\hline $\mathrm{C}$ & 24 & 13 & 23 & 12 & 65 & $z=2.60, p<.01$ \\
\hline $\mathrm{D}$ & 21 & 9 & 27 & 15 & 67 & $z=2.84, p<.01$ \\
\hline I & 11 & 4 & 32 & 25 & 60 & $z=1.65, p>.05$ \\
\hline
\end{tabular}


ing into a coherent representation of a compound stimulus as a functionally distinct stimulus that is, nevertheless, lawfully related to its components" (p. 351). For example, Chase and Heinemann (1972) were able to account for the improved discriminability of compounds, as opposed to elements, by treating the sensations produced by the compound as a bivariate distribution from which variations along the component dimensions produced smaller changes in behavior than their combined effect. George and Pearce (2003) have provided convincing evidence that with simultaneous training, pigeons learn not only about form and color, but also about the location of stimuli associated with reward or nonreward, a process they refer to as feature binding. The present experiments provide evidence that, in the absence of simultaneous training, feature binding does not occur. Training on alternate days, with the elements experienced as a compound only on test trials, appears to have resulted in separate associations between the elements on each dimension and the choice keys.

These experiments also provide important theoretical information about how visual information is processed. There is clear evidence that pigeons can categorize complex visual patterns, discriminating, for example, between paintings by different artists (Watanabe, Sakamoto, \& Wakita, 1995), or, in the classic experiment of Herrnstein and Loveland (1964), photographs with or without a person. Because such stimuli cannot be categorized according to a single set of features, it has been suggested that individual features become associated with each category. This makes it possible for new exemplars that contain some or all of the features to be categorized correctly. Quantitative tests of such feature models are made possible by simplifying the stimuli-for example, by using line drawings in which the features can be described in terms of sets of geometric primitives, called geons (e.g., Van Hamme, Wasserman, \& Biederman, 1992), or by establishing probabilistic associations between predefined features and the categories associated with reward or nonreward (e.g., Jistumori, 1993; Lea \& Ryan, 1990).

The natural intelligence model (NIM) of Heinemann and Chase (1990) and Chase and Heinemann (2001) and the instance model of D. S. Blough (1998) provide an alternative to feature models of categorization. Increasing evidence in both the animal literature (e.g., Cook, Levison, Gillett, \& Blaisdell, 2005; Vaughan \& Greene, 1984) and the human literature (e.g., Erickson \& Kruschke, 1998; Logan, 1988; Nosofsky, 1989) that individual instances are preserved in memory makes exemplar models particularly promising. According to NIM and the instance model of Blough events are preserved intact as individual records in exemplar memory, a relatively long-term but limited-capacity memory. Each record contains information about the sensation experienced and the response that is reinforced. When a decision is required, the subject decides which response will result in delivery of the reinforcer. According to these models, this is accomplished by comparing the sensation produced by the stimulus to be identified with a small sample of records drawn from exemplar memory. The choice made is the one most likely to be correct, given the information in the sample. Accuracy is a function of the difference in the sensations associated with the discriminative stimuli and the number of records sampled - the larger the differences in the sensations and the larger the number of records, the fewer the errors.

Simulations using NIM, in which it was assumed that 10 records were drawn randomly from exemplar memory, enabled us to compare the difference in accuracy expected if both color and form were used on compatible test trials with that expected on training trials in which information was provided by only color or only form; with sensitivity to stimulus differences comparable to that found in the present experiments, accuracy would be about $94 \%$ on test trials and $86 \%$ on training trials. This difference reflects use of all 10 records when the stimulus to be identified provides both color and form information (compatible test trials) as opposed to 5 useful records when the stimulus to be identified is only a color or a form (training trials). Thus, this theoretical analysis provides further evidence that retrieval of information from exemplar memory is random and that, when elements from both dimensions are presented together, pigeons attend to only one of the elements if trained separately on form and color.

NIM has been applied successfully to more complex categorization tasks (Donis, Chase, \& Heinemann, 2005; Donis \& Heinemann, 1993; Van Hamme et al., 1992) than those used here. Details of how NIM works for simple dot matrix patterns such as those used by Donis and Heinemann are available in Chase and Heinemann (2001) along with a computer program that can be used for simple simulations. The challenge remains to precisely describe the processes through which pigeons, with apparent ease, process the information in the complex and ever changing visual world. Whether exemplar or feature models, a combination of both, or yet another type of model will provide the answer awaits further research. The present research suggests that the answer lies in the structure of the training task.

\section{REFERENCES}

Blough, D. S. (1996). Error factors in pigeon discrimination and delayed matching. Journal of Experimental Psychology: Animal Behavior Processes, 22, 118-131.

Blough, D. S. (1998). Context reinforcement degrades discriminative control: A memory approach. Journal of Experimental Psychology: Animal Behavior Processes, 24, 185-199.

Blough, D. S. (2002). Measuring the search image: Expectation, detection, and recognition in pigeon visual search. Journal of Experimental Psychology: Animal Behavior Processes, 28, 397-405.

Blough, P. M. (1991). Selective attention and search images in pigeons. Journal of Experimental Psychology: Animal Behavior Processes, 17, 292-298.

Brown, M. F., \& Morrison, S. K. (1990). Element and compound matching-to-sample performance in pigeons: The roles of information load and training history. Journal of Experimental Psychology: Animal Behavior Processes, 16, 185-192.

Chase, S., \& Heinemann, E. G. (1972). Choices based on redundant information: An analysis of two-dimensional stimulus control. Journal of Experimental Psychology, 92, 161-175.

Chase, S., \& Heinemann, E. G. (2001). Exemplar memory and discrimination. In R. G. Cook (Ed.), Avian visual cognition [Online]. Retrieved Dec. 23, 2005, from www.pigeon.psy.tufts.edu/avc/chase/. 
Cook, R. G., Levison, D. G., Gillett, S. R., \& Blaisdell, A. P. (2005). Capacity and limits of associative memory in pigeons. Psychonomic Bulletin \& Review, 12, 350-358.

CooK, R. G., \& Wixted, J. T. (1997). Same-different texture discrimination in pigeons: Testing competing models of discrimination and stimulus integration. Journal of Experimental Psychology: Animal Behavior Processes, 23, 401-416.

Donis, F. J., Chase, S., \& Heinemann, E. G. (2005). Effects of identical context on visual pattern recognition by pigeons. Learning \& Behavior, 33, 90-98.

Donis, F. J., \& Heinemann, E. G. (1993). The object-line inferiority effect in pigeons. Perception \& Psychophysics, 53, 117-122.

Emmerton, J. (2001). Birds' judgments of number and quantity. In R. G. Cook (Ed.), Avian visual cognition [Online]. Retrieved Dec. 23, 2005, from www.pigeon.psy.tufts.edu/avc/emmerton/.

Erickson, M. A., \& KruschKe, J. K. (1998). Rules and exemplars in category learning. Journal of Experimental Psychology: General, 127, 107-140.

George, D. N., \& Pearce, J. M. (2003). Discrimination of structure: II. Feature binding. Journal of Experimental Psychology: Animal Behavior Processes, 29, 107-117.

Grant, D. S., \& MacDonald, S. E. (1986). Matching to element and compound samples in pigeons: The role of sample coding. Journal of Experimental Psychology: Animal Behavior Processes, 12, 160-171.

Heinemann, E. G., \& Chase, S. (1990). A quantitative model for pattern recognition. In M. L. Commons, R. J. Herrnstein, S. M. Kosslyn, \& D. B. Mumford (Eds.), Quantitative analyses of behavior: Vol. 9. Computational and clinical approaches to pattern recognition and concept formation (pp. 109-126). Hillsdale, NJ: Erlbaum.

Herrnstein, R. J., \& Loveland, D. H. (1964). Complex visual concept in the pigeon. Science, 146, 549-551.

Honig, W. K., \& STEWART, K. E. (1989). Discrimination of relative numerosity by pigeons. Animal Learning \& Behavior, 17, 134-146.

JiTSUMORI, M. (1993). Category discrimination of artificial polymorphous stimuli based on feature learning. Journal of Experimental Psychology: Animal Behavior Processes, 19, 244-254.

Kehoe, E. J., \& Gormezano, I. (1980). Configuration and combination laws in conditioning with compound stimuli. Psychological Bulletin, 87, 351-378.

LANGley, C. M., \& Riley, D. A. (1993). Limited capacity information processing and pigeon matching-to-sample: Testing alternative hypotheses. Animal Learning \& Behavior, 21, 226-232.

LEA, S. E. G., \& Ryan, C. M. E. (1990). Unnatural concepts and the theory of concept discrimination in birds. In M. L. Commons, R. J. Herrnstein, S. M. Kosslyn, \& D. B. Mumford (Eds.), Quantitative analyses of behavior: Vol. 8. Behavioral approaches to pattern recognition and concept formation (pp. 164-185). Hillsdale, NJ: Erlbaum.

Logan, G. D. (1988). Toward an instance theory of automatization. Psychological Review, 95, 492-527.

NosofsKy, R. M. (1989). Further tests of an exemplar-similarity approach to relating identification and categorization. Perception \& Psychophysics, 45, 279-290.

Pietrewicz, A. T., \& Kamil, A. C. (1979). Search image formation in the blue jay (Cyanocitta cristata). Science, 204, 1332-1333.

Roberts, W. A., \& Grant, D. S. (1978). Interaction of sample and comparison stimuli in delayed matching to sample with the pigeon. Journal of Experimental Psychology: Animal Behavior Processes, 4, 68-82.

SCHUPAK, C. (2002). Information processing during the presolution period: Stimulus location effects in discrimination learning (Doctoral dissertation, City University of New York, 2002). Dissertation Abstracts International, 63(3-B), 1604.

Van Hamme, L. J., Wasserman, E. A., \& Biederman, I. (1992). Discrimination of contour-deleted images by pigeons. Journal of Experimental Psychology: Animal Behavior Processes, 18, 387-399.

Vaughan, W., \& Greene, S. L. (1984). Pigeon visual memory capacity. Journal of Experimental Psychology, 10, 256-271.

Watanabe, S., SaKamoto, J., \& Wakita, M. (1995). Pigeons' discrimination of paintings by Monet and Picasso. Journal of the Experimental Analysis of Behavior, 63, 165-174.

(Manuscript received July 27, 2005; revision accepted for publication December 19, 2005.) 